\title{
HIV-1 protease inhibitors and cytomegalovirus vMIA induce mitochondrial fragmentation without triggering apoptosis
}

\author{
Cell Death and Differentiation (2006) 13, 348-351. doi:10.1038/sj.cdd.4401750; published online 26 August 2005
}

\section{Dear Editor,}

Recent studies suggest that mitochondrial dynamics (fission and fusion) has a major impact on the regulation of mitochondrial outer membrane permeabilization, ${ }^{1,2}$ which in turn determines the point-of-no-return of apoptotic and necrotic cell death. ${ }^{3}$ Thus, programmed cell death, as it occurs in Caenorhabditis elegans development, is accompanied by mitochondrial fragmentation. Overexpression of a dominant-negative (DN) mutant of dynamin-related protein-1 (DRP-1), which inhibits mitochondrial fission, can reduce the incidence of cell death induced by a $\mathrm{BH} 3-$ only protein in vivo, in C. elegans. ${ }^{4}$ These data confirm prior observations obtained in vitro, in mammalian cell culture systems, in which a DN point mutant of human DRP-1 (DRP-1 $\left.{ }^{\mathrm{K} 38 \mathrm{~A}}\right)^{5}$ was found to inhibit mitochondrial fragmentation and cell death induced by different apoptosis inducers. ${ }^{6}$ Conversely, the overexpression of wild-type DRP-1, which causes extensive mitochondrial fragmentation without cell death, can inhibit apoptosis induction by ceramide in tumor cells, presumably by reducing the transmission of calcium waves through the cytoplasm. ${ }^{7}$

Mitochondrial fragmentation has been previously reported to affect the organs of HIV-1 carriers subjected to highly active antiretroviral therapy (HAART) and in particular to treatment with HIV-specific protease inhibitors. ${ }^{8,9}$ Since some HIV-1specific protease inhibitors such as ritonavir (Rtv) and nelfinavir (Nfv) can modulate apoptosis in vitro ${ }^{10}$ and in vivo, in mouse models of fulminant hepatitis, septic shock and temporary carotid occlusion, ${ }^{11}$ we investigated whether such protease inhibitors would regulate cell death via an effect on mitochondrial dynamics. To this end, we took advantage of syncytia, which due to their oversize offer a considerable advantage for light microscopic examination. ${ }^{12}$ Syncytia were generated by the fusion of HeLa cells expressing CD4 and HeLa cells expressing the HIV-1 envelope, ${ }^{13}$ then exposed to HIV-1 protease inhibitors at therapeutically achievable concentrations $^{14}$ and monitored for mitochondrial morphology. Nfv, Rtv (Figure 1) and saquinavir (not shown) caused a marked fragmentation of the mitochondrial network into discrete, punctuate cytoplasmic dots, and this effect was partially inhibited by prior transfection with $\mathrm{DRP}-1^{\mathrm{K} 38 \mathrm{~A}}$ (Labrousse et al. ${ }^{5}$ ) (Figure 1a, b). Similarly, the knockdown of hFis1, another gene involved in nuclear fission, ${ }^{15}$ has a partial, yet highly significant inhibitory effect on mitochondrial fission induced by Nfv or Rtv (Figure 1b), underscoring that it involved specific (rather than nonspecific toxic) effects on mitochondrial dynamics. Thus, Nfv and Rtv can affect mitochondrial dynamics in a virus-free system, which lacks the HIV-1 protease. Importantly, Nfv and Rtv enforced mitochondrial fragmentation in conditions in which cytochrome $c$ continued to be present in mitochondria and hence to colocalize with the mitochondrial marker Hsp60, as determined by two-color immunofluorescence (Figure 1a and b). HIV-1 Env-elicited syncytia undergo p53-dependent cell death after a latency period, ${ }^{16}$ as detectable by FACS analysis, ${ }^{17}$ and this phenomenon was not affected by Nfv or Rtv (Figure 1c). Moreover, Nfv or Rtv had no (positive or negative) effect on apoptosis induction induced by a CD95agonistic antibody (7C11), staurosporine (STS) or acetylceramide (C2), as determined by FACS analysis of the mitochondrial transmembrane potential and cell viability ${ }^{18}$ (Figure 1d). In contrast to mitochondrial uncouplers, which can induce mitochondrial fragmentation while negatively affecting the mitochondrial transmembrane potential $\left(\Delta \Psi_{\mathrm{m}}\right),{ }^{19,20} \mathrm{Nfv}$ or Rtv did not dissipate the $\Delta \Psi_{\mathrm{m}}$ (Figure 1d). Thus, a dramatic fragmentation of the mitochondrial network failed to induce apoptosis and even failed to modulate (facilitate or inhibit) apoptosis induction by endogenous or exogenous stimuli.

Apoptotic stimuli, like $\mathrm{C}_{2}$ ceramide and $\mathrm{H}_{2} \mathrm{O}_{2}$, induce $\mathrm{Ca}^{2+}$ release from the endoplasmic reticulum (ER), ${ }^{21}$ leading to $\mathrm{Ca}^{2+}$ uptake into the mitochondrial network. This process is initially limited to restricted areas (G Szabadkai, D De Stefani, $\mathrm{R}$ Rizzuto, unpublished), but then leads to mitochondrial $\mathrm{Ca}^{2+}$ overload, loss of mitochondrial membrane potential and release of proapoptotic factors (such as cytochrome $c$, AIF, or Omi/HtrA2) from the intermembrane space (for recent reviews see Garrido and Kroemer, ${ }^{22}$ and Danial and Korsmeyer ${ }^{23}$ ). Similarly, inositol 1,4,5-trisphosphate $\left(\mathrm{IP}_{3}\right)$-induced $\mathrm{Ca}^{2+}$ release leads to localized mitochondrial $\mathrm{Ca}^{2+}$ uptake, followed by intramitochondrial $\mathrm{Ca}^{2+}$ diffusion, resulting in complete $\mathrm{Ca}^{2+}$ charge of the entire network. ${ }^{7,24}$ Division of the continuous mitochondrial network by Drp-1 (see above) disrupts these intramitochondrial $\mathrm{Ca}^{2+}$ waves, thus impeding the full mitochondrial $\mathrm{Ca}^{2+}$ upload. Importantly, reduced mitochondrial $\mathrm{Ca}^{2+}$ accumulation of the fragmented mitochondrial network has a protective role against $\mathrm{Ca}^{2+}$ mediated mitochondrial outer membrane permeabilization and apoptosis. ${ }^{1,7}$ A prerequisite for this effect is that during mitochondrial division no change in the overall mitochondrial 
a

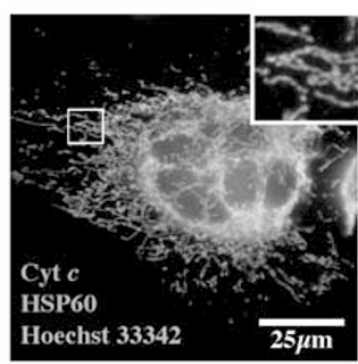

Nelfinavir

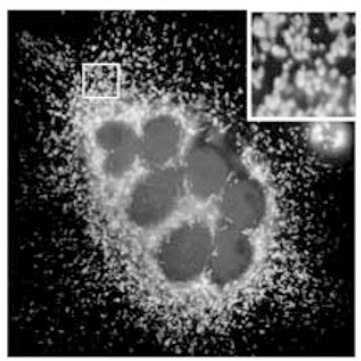

Nelfinavir+Drp-1 ${ }^{\text {K38 A }}$

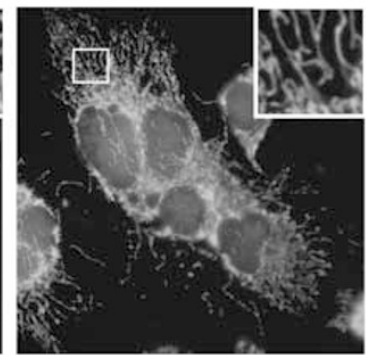

b
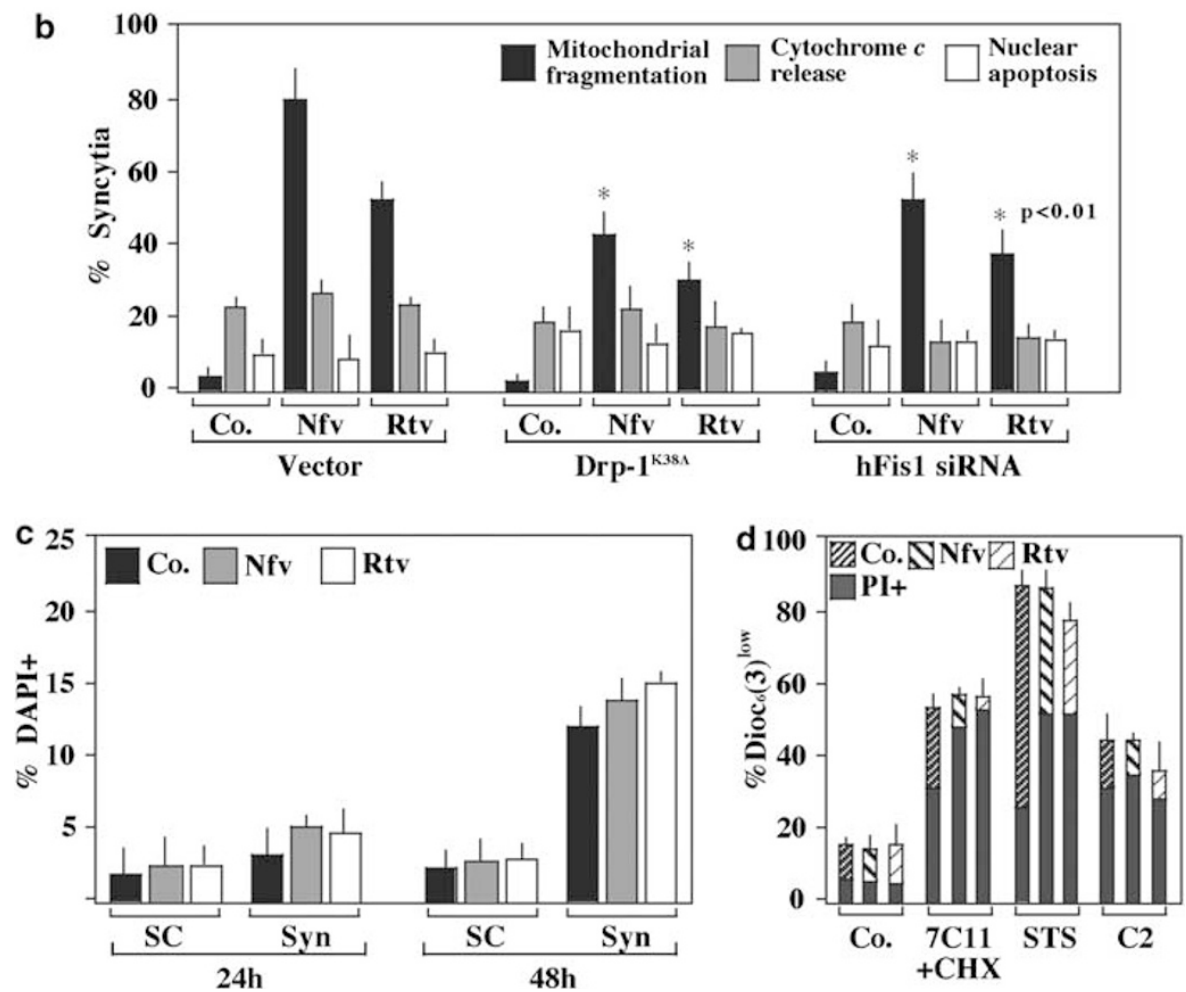

Figure 1 Effect of HIV protease inhibitors on mitochondrial dynamics and apoptosis susceptibility. (a, b) Effect of Nelfinavir (Nfv) or Ritonavir (Rtv). HeLa cells expressing CD4 and HeLa cells transfected with HIV-1 Env ${ }^{\mathrm{LAI}}$ were transfected with vector only (left and middle panels in a), with a dominant-negative (DN) DRP mutant (DRP-1 ${ }^{\mathrm{K} 38 \mathrm{~A}}$ right panel in a), or with pSuper vectors designed to knockdown $\mathrm{hFis1}$, (James et al. ${ }^{15}$ ) then cocultured to form syncytia (Castedo et al. ${ }^{13}$ ), treated for $24 \mathrm{~h}$ with Nfv (Agouron) and Rtv (Abbott) (both at $10 \mu \mathrm{M}$ ), fixed with paraformaldehyde, and subjected to a triple staining with a polyclonal antibody specific for Cyt $c$ (Santa Cruz), a monoclonal antibody specific for heat shock protein (HSP) 60 (Sigma), suitable secondary Alexa conjugates, and the chromatin stain Hoechst 33342 (Molecular Probes). Note the yellow overlap between the green and red fluorescence (a), indicating mitochondrial retention of Cyt $c$. The inserts in the upper right corner correspond to the marked rectangles in each microphotograph in (a). The statistics of mitochondrial fragmentation, Cyt $c$ release and nuclear chromatin condensation induced by Nfv and Rtv are shown in (b). Asterisks denote significant inhibitory effects of DRP-1 ${ }^{\mathrm{K} 38 \mathrm{~A}}$ or $\mathrm{hFis} 1$ siRNA on the Nfv- or Rtv-induced mitochondrial fragmentation $(P<0.01$, paired Student's $t$-test). The transfection efficiency, as estimated by transfection with EGFP, was estimated to be $\sim 70 \%$. (c) Spontaneous cell death of syncytia treated with Nfv or Rtv. HeLa CD4 and HeLa Env cells were prestained with Cell Tracker red and green, respectively, and then cocultured, meaning that syncytia could be identified as double-positive red/green cells. The frequency of cells whose plasma membrane became permeable to $4^{\prime}, 6$-diamidino-2-phenylindole dihydrochloride (DAPI) was determined by cytofluorometry (Roumier et al. ${ }^{11}$ ), either after 24 or $48 \mathrm{~h}$ of coculture. (d) Effect of Nfv or Rtv on apoptosis induction by the CD95 agonistic antibody 7C11 $(1 \mu \mathrm{g} / \mathrm{ml}$, Immunotech) plus cycloheximide (CHX, $3.5 \mu \mathrm{M})$, staurosporine (STS, $200 \mathrm{nM})$ or acetylceramide $(\mathrm{C} 2,40 \mu \mathrm{M}$, Sigma). HeLa cells were pretreated for $24 \mathrm{~h}$ with Nfv or Rtv (both at $10 \mu \mathrm{M}$ ) and then treated with the indicated agents for $18 \mathrm{~h}$. The percentage of dying cells with a low mitochondrial transmembrane potential was determined using 3,3' dihexyloxacarbocyanine iodide (DiOC6(3) and that of dead cells was determined using the vital dye propidum iodine (PI), using published methods (Castedo et al. ${ }^{18}$ ). All experiments were repeated at least two times, yielding similar results

volume, subcellular localization and/or colocalization with the $\mathrm{ER} \mathrm{Ca}^{2+}$ release sites occurs. Indeed, the overexpression of hFis1 in HeLa cells, apart from causing massive breakdown of the mitochondrial network, can also lead to perinuclear clustering of mitochondrial fragments. Parallel with these changes, no reduction in mitochondrial $\mathrm{Ca}^{2+}$ uptake could be observed following $\mathrm{IP}_{3}$-induced $\mathrm{Ca}^{2+}$ release in these cells, ${ }^{25}$ and importantly cells overexpressing hFis1 were more susceptible for apoptosis. Along these lines, using mitochondrially targeted recombinant $\mathrm{Ca}^{2+}$ sensitive probes, we assessed the overall and local $\mathrm{Ca}^{2+}$ mitochondrial signaling following $\mathrm{IP}_{3}$-mediated $\mathrm{Ca}^{2+}$ release in $\mathrm{Nfv}$ - and Rtv-treated 

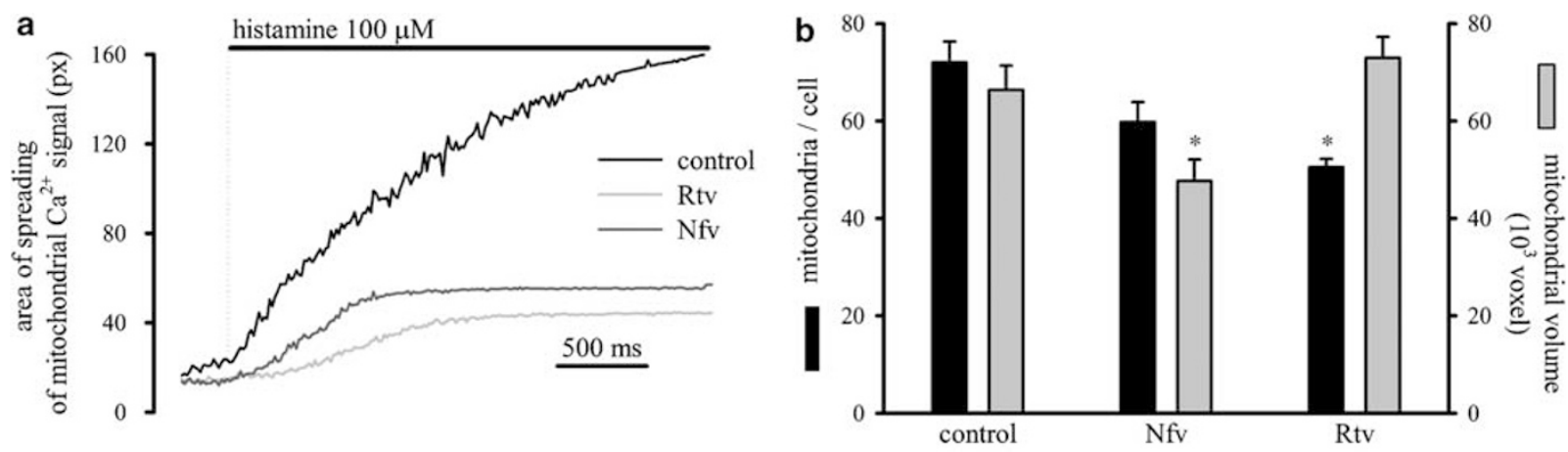

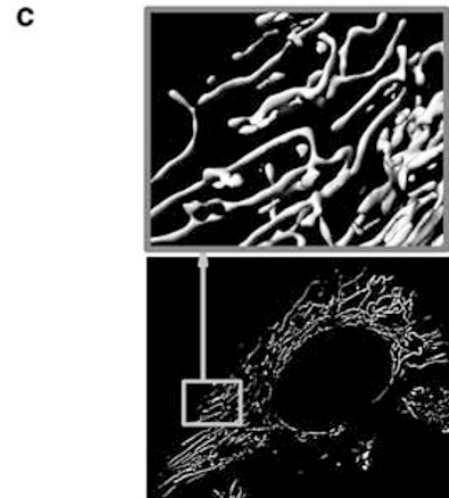

control

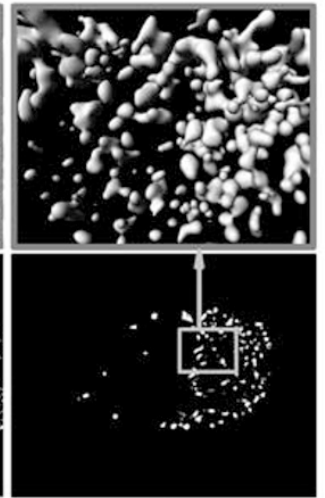

Nfv

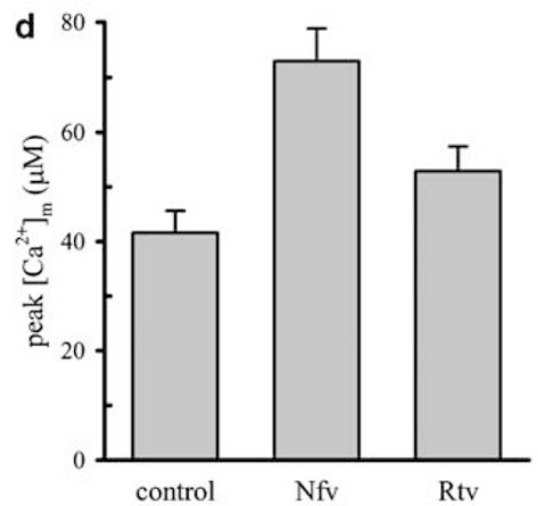

Figure 2 Relationship between mitochondrial morphology and $\mathrm{Ca}^{2+}$ homeostasis in Nfv- and Rtv-treated cells. Mitochondrial $\mathrm{Ca}^{2+}$ signals were measured in single cells (a) and cell population (c) using the mitochondrion-targeted $\mathrm{Ca}^{2+}$ probes $2 \mathrm{mtRP}$ (Bianchi et al. ${ }^{33}$ ) and mtAEQmut (Chiesa et al. ${ }^{34}$ ), respectively. (a) Spreading of $\mathrm{Ca}^{2+}$ signal was quantified as the area of fast $\mathrm{Ca}^{2+}$ diffusion measured on time-derived images of $2 \mathrm{mtRP}$ time lapse series of images. Acquisition and analysis of images was performed using the Meta Imaging Series 5.0 software package (Universal Imaging) as described in Szabadkai et al. ${ }^{7}$ and Gerencse and Adam-Vizi ${ }^{24}$. The traces shown are mean of 15-18 cells from three different preparations. (b, c) The number and volume of individual continuous mitochondrial objects was quantified on mtGFP transfected HeLa cells (controls) and after treatment with Nfv and Rtv (12-16 h, $10 \mu \mathrm{M}$ both). Z-series of images were acquired using a digital microscopy system as described in Szabadkai et al. ${ }^{7}$. Images were deconvoluted with the custom-made EPR software (Carrington et al. ${ }^{35}$ ). One deconvoluted image plane of control and Nfv-treated cells are shown on $\mathbf{c}$ (lower panel). 3D reconstruction was performed either by volume (using the custom-made software DAVE, Carrington et al. ${ }^{35}$ ) or surface rendering (Imaris 4.1, Bitplane AG, trial version, upper panels), object definition/counting and quantitative volume analysis was performed by DAVE (b) The results are mean ( \pm S.E.M.) of $12-14$ cells from two different preparations. $\left({ }^{\star} P<0.05\right.$, Student's $t$-test) (d) Overall $\left[\mathrm{Ca}^{2+}\right]_{\mathrm{m}}$ responses of HeLa cells to $100 \mu \mathrm{M}$ histamine. Histamine induces sustained $\mathrm{IP}_{3}$-mediated $\mathrm{Ca}^{2+}$ release and consequent $\mathrm{Ca}^{2+}$ accumulation in mitochondria. ${ }^{7}\left[\mathrm{Ca}^{2+}\right]_{\mathrm{m}}$ peaks $($ mean \pm S.E.M.), measured on a population of $\sim 200000$ cells transfected transiently with the mtAEQmut (Chiesa et al..$^{34}$ ) probe (controls) and after Nfv and Rtv treatment (10 $\mu \mathrm{M}, 12-$ $16 \mathrm{~h}$ ), are shown in at least 15 experiments from four different preparations

HeLa cells. Division of the mitochondrial network induced by the HIV protease inhibitors led to a drastic reduction of intramitochondrial spreading of the $\mathrm{Ca}^{2+}$ signal (Figure 2a), as measured in single HeLa cells transfected with the mitochondrially targeted ratiometric pericam probe 2mtRP. ${ }^{7,26}$ However, at variance with Drp-1-induced forced mitochondrial division, ${ }^{7}$ morphometric analysis of mtGFPexpressing Nfv- and Rtv- treated HeLa cells showed not only the fragmentation of the mitochondrial network, but also two other important morphological features: (i) fragmented mitochondrial particles were found to be clustered perinuclearly in both Nfv- and Rtv-treated cells (Figure 2c), and (ii) quantitative volume analysis revealed a significant decrease in mitochondrial number and volume for Rtv- and Nfv-treated cells, respectively, within the technical limitations of confocal microscropy (Figure 2b). A plausible consequence of these changes is the increase of ER-mitochondrial contacts relative to the mitochondrial volume, since the ER has a higher density in the perinuclear region. Indeed, population measurement in
HeLa cells, transfected with the mitochondrially targeted lowaffinity mutant aequorin (mtAEQmut) showed that the overall mitochondrial $\mathrm{Ca}^{2+}$ accumulation in $\mathrm{Nfv}$ - and Rtv-treated cells is more efficient, leading to higher $\left[\mathrm{Ca}^{2+}\right]$ peak values during histamine-induced $\mathrm{Ca}^{2+}$ signals (Figure 2d). These findings explain why the compounds do not have protective effect against $\mathrm{Ca}^{2+}$-dependent apoptotic stimuli, and provide further insight into the mechanisms through which mitochondrial morphology may determine cellular fate in stress conditions.

In a further series of experiments, we investigated the effect of the cytomegalovirus early gene product viral mitochondrial inhibitor of apoptosis (VMIA) 27,28 (a product of the UL37 exon1 gene) on mitochondrial morphology. VMIA induced a marked shift from a tubular to a punctuate mitochondrial distribution (McCormick et al. ${ }^{29}$ and Supplementary Figure 1a), as detectable in normal HeLa cells. ${ }^{29}$ The vMIA-driven disintegration of the mitochondrial network was not modulated by transfection with DRP-1 ${ }^{\mathrm{K} 38 \mathrm{~A}}$ nor by knockdown of hFis1 
(Supplementary Figure 1b), although these genetic manipulations did have a significant impact on Nfv- or Rtv-induced mitochondrial fragmentation (Figure 1b). Moreover, DRP$1^{\mathrm{K} 38 \mathrm{~A}}$ or hFis 1 knockdown did not antagonize the capacity of VMIA to inhibit apoptosis induction by CD95 activation (with 7C11) or general tyrosine kinase inhibition (with STS) (Supplementary Figure 1c). Thus, mitochondrial fragmentation mediated by VMIA is accompanied by strong apoptosis inhibition, and both phenomena are not affected by DRP. $1^{\mathrm{K} 38 \mathrm{~A}}$ or hFis1.

As a result, we are confronted with two situations in which mitochondrial fragmentation does not lead to an enhanced mitochondrial permeabilization. In the first case, HIV-1 protease inhibitors reduce the connectivity of the mitochondrial network in a fashion that involves proteins of the fission/ fusion machinery (such as Drp-1 and Fis1). In the second case, VMIA causes mitochondrial fragmentation through a different, yet to be elucidated pathway. However, in both cases reported here, mitochondrial fragmentation fails to induce apoptosis or to facilitate its induction, as this has been also reported for the simultaneous knockdown of hFis 1 and Opa1. ${ }^{30}$ HIV-1 protease inhibitors were inert as far as cell death induction is concerned (Figure 1), although it has been reported that modulation of mitochondrial connectivity by Drp1 and DRP $-1^{\mathrm{K} 38 \mathrm{~A}}$ protects cells against ceramide and STS, respectively. ${ }^{6,7}$ VMIA, which induces marked mitochondrial fragmentation, is - paradoxically - a rather potent, general inhibitor of MOMP and apoptosis. ${ }^{27,28,31}$ vMIA inhibits MOMP, presumably by recruiting Bax to mitochondria while antagonizing its pore-forming function. ${ }^{28,32}$ However, vMIA can induce mitochondrial fragmentation in cells that lack Bax expression (not shown), indicating that the precise mechanism through which VMIA influences mitochondrial dynamics are elusive.

Irrespective of these incognita, however, it appears clear that mitochondrial fission is not automatically linked to apoptosis induction and that this link, if it exists, must be indirect and depends on cell types, the specific modulator of mitochondrial dynamics and/or the specific cell death inducer.

\section{Acknowledgements}

We thank Dr. AM van der Bliek (University of California Los Angeles, CA, USA) and JC Martinou (University of Geneva, Switzerland) for expression and siRNA vectors, respectively. This work has been supported by a special grant from the Ligue Nationale contre le Cancer, grants from ANRS and the European Commission (to GK), Telethon-Italy (Grants no. 1285 and GTF02013), the Italian Association for Cancer Research (AIRC), the Human Frontier Science Program, the Italian University Ministry (MURST and FIRB) and the Italian Space Agency (ASI) (to RR).

\section{T Roumier ${ }^{1}$, G Szabadkai', A-M Simoni', J-L Perfettini',} A-L Paulau ${ }^{1}, M$ Castedo $^{1}, D$ Métivier ${ }^{1}$, A Badley $^{3}$, $R$ Rizzuto $^{2}$ and $G$ Kroemer,

1 CNRS-UMR8125, Laboratoire de Génomique Cellulaire des Cancers, Institut Gustave Roussy, 39 rue Camille-Desmoulins, F-94805 Villejuif, France

2 Department of Experimental and Diagnostic Medicine, Section of General Pathology, and Interdisciplinary Center for the Study of Inflammation (ICSI), University of Ferrara, Ferrara 44100, Italy

3 Division of Infectious Diseases, Mayo Clinic, Rochester, MN 55905, USA

* Corresponding author: G Kroemer, CNRS-UMR8125, Institut Gustave Roussy, Pavillon de Recherche 1, 39 rue Camille-Desmoulins, F-94805 Villejuif, France. Tel: + 33-1-42 1160 46; Fax: + 33-1-42 1160 47;

E-mail: kroemer@igr.fr

1. Perfettini J-L et al. (2005) Trends Cell Biol. 15: 179-183

2. Lucken-Ardjomande S and Martinou JC (2005) J. Cell Sci. 118: 473-483

3. Green DR and Kroemer G (2004) Science 305: 626-629

4. Jagasia $R$ et al. (2005) Nature 433: 754-760

5. Labrousse AM et al. (1999) Mol. Cell. 4: 815-826

6. Frank S (2001) Dev. Cell 1: 515-525

7. Szabadkai G et al. (2004) Mol. Cell. 8: 59-68

8. Tolomeo M et al. (2003) J. Clin. Pathol. 56: 147-151

9. Verucchi $G$ et al. (2004) J. Acquir. Immune Defic. Syndr. 35: 326-328

10. Phenix BN (2001) Blood 98: 1078-1085

11. Weaver JGR et al. (2005) J. Clin. Invest. 115: 1828-1938

12. Castedo M and Kroemer G. (2002) Trends Cell Biol. 12: 446-447

13. Castedo $\mathrm{M}$ et al. (2002) EMBO J. 21: 4070-4080

14. Badley AD et al. (2003) Trends Pharmacol Sci. 24: 298-305

15. James DI et al. (2003) J. Biol. Chem. 278: 36373-36379

16. Boya $P$ et al. (2005) Mol. Cell. Biol. 25: 1025-1040

17. Roumier T et al. (2003) Biochem. Pharmacol. 66: 1321-1329

18. Castedo $\mathrm{M}$ et al. (2002) J. Immunol. Methods 265: 39-47

19. Legros $\mathrm{F}$ et al. (2002) Mol. Biol. Cell. 13: 4343-4354

20. Lyamzaev KG et al. (2004) Biochem. Soc. Trans. 32: 1070-1071

21. Pinton $P$ et al. (2001) EMBO J. 20: 2690-2701

22. Garrido C. and Kroemer G (2004) Curr. Opin. Cell Biol. 16: 639-646

23. Danial NN and Korsmeyer S. (2004) Cell 116: 205-219

24. Gerencse AA and Adam-Vizi V (2005) Biophys. J. 88: 698-714

25. Frieden $\mathrm{M}$ et al. (2004) J. Biol. Chem. 279: 22704-22714

26. Filippin L et al. (2003) J. Biol. Chem. 278: 39224-39234

27. Goldmacher VS et al. (1999) Proc. Natl. Acad. Sci. USA 96: 12536-12541

28. Poncet $D$ et al. (2004) J. Biol. Chem. 279: 22605-22614

29. McCormick AL et al. (2003) J. Virol. 77: 631-641

30. Lee YL et al. (2004) Mol. Biol. Cell. 15: 5001-5011

31. Castedo M et al. (2004) Oncogene 23: 4362-4370

32. Arnoult D et al. (2004) Proc. Natl. Acad. Sci. USA 101: 7988-7993

33. Bianchi K et al. (2004) Biochim. Biophys. Acta 1742: 119-131

34. Chiesa A et al. (2001) Biochem. J. 355: 1-12

35. Carrington WA et al. (1995) Science 268: 1483-1487

Supplementary Information accompanies the paper on Cell Death and Differentiation website (http://www.nature.com/cdd) 